in a variety of ways. The tool should increase staff knowledge of specific medications, increase confidence, provide staff with the ability to challenge or recommend such medications in order to provide person-centred care and be available for continuing professional development and revalidation. It should be easily accessible, 'bite-size' to encourage use and available via various sources - email, notice boards, face to face delivery.

Methods Drugs were identified through drug incidents and staff feedback. From this newsletters were generated in a standard one-sided A4 format to provide information.

Results To date 6 newsletters have been produced to include; Ketamine, Phenobarbital, Ketorolac, NOACs, Dexamethasone and Levetiracetam. All follow the same format listing indications, doses, side-effects, contra-indications, interactions, administration advice and supply issues. The newsletter also include feedback and updates regarding medicines management, stock information and short quizzes to increase costeffective use of medicines.

Conclusion all members of the team have found the newsletter beneficial, in particular knowledge and confidence in the use of Phenobarbital has increased. To increase awareness newsletters will be presented in medical and nursing inductions. We will continue to seek feedback for future drugs on concerns and challenges faced and pre-empt new drugs on the market. The Medicines Management champions will take ownership and develop future newsletters in conjunction with the pharmacist. A questionnaire will be circulated to identify what improvements can be made to the newsletter.

\section{IN HOUSE TEACHING OF COMMUNICATION SKILLS USING EXPERIENTIAL TRAINING FOR HOSPICE STAFF AT A HOSPICE IN BIRMINGHAM}

Brenda Ward, Sarah Bache, Philippa Olliver. John Taylor Hospice, Birmingham

\subsection{6/bmjspcare-2018-ASPabstracts.36}

Project aim To provide a safe space for the experiential learning and practising of communication skills for hospice professionals.

Method A series of one hour workshops for up to 10 trained health care professionals were carried out at the hospice over a 7 month period (March to September 2017) facilitated by the hospice consultant and consultant nurse. Using a modified version of the 'fish bowel technique', a Clinical Nurse Specialist in palliative care was used as the actress. For each workshop, the participants discussed from their experiences and decided on their own challenging topic to practice their skills. Communication was discussed as the consultation progressed with both the facilitators and the actress giving feedback. Participants were encouraged to reflect on what they had heard from others. Participant written feedback was sought immediately after the workshop and in addition delayed feedback to determine the usefulness of the sessions to staff practice after the event.

Results 16 Health Care professionals attended the sessions including a doctor, nurses from the inpatient unit, the community and day hospice. In addition, a visiting medical student and 2 student nurses participated. Topics included Advance Care Planning, CPR discussions, talking with a very depressed patient, answering 'Am I dying?' Several staff attended more than one workshop, in these the facilitators saw definite improvements in their communication skills. Feedback, both immediate and delayed was extremely positive. All participants, ranging from students to experienced practitioners requested further opportunities to practice in this manner. The majority in each workshop stated they were more likely to broach the difficult topic and had learned skills to assist them.

Conclusion This simple method of teaching is a highly appreciated, successful method of training hospice staff in honing their communication skills and is suitable for professionals with a range of professional experience.

\section{DEVELOPING GUIDELINES FOR OPIOID PRESCRIBING AND ADJUSTMENT IN RENAL IMPAIRMENT IN AN ACUTE MEDICAL ADMISSIONS UNIT}

Kate Howorth, Emma Foggett, Jane Atkinson, Fraser Henderson, Eleanor Grogan, Katie Frew. Northumbria Healthcare NHS Foundation Trust

\subsection{6/bmjspcare-2018-ASPabstracts.37}

Background Incorrect opioid prescribing can significantly impact patient care and safety, resulting in poor pain control or risk of opioid toxicity. Renal failure often means adjustment of opioid doses is needed. Local audit demonstrated incorrect prescribing in acute medical admissions, particularly in patients with renal impairment, and a survey showed low confidence amongst junior doctors in prescribing opioids.

Methods Local specific guidelines were developed for opioid prescribing and adjustment in Acute Kidney Injury (AKI) and for initiating opioids in patients with a reduced eGFR on the medical admissions unit. These were in line with the North of England Cancer Network Palliative Care Guidelines and were reviewed by the Trust's Palliative Care team. Teaching was conducted with the junior doctors working in acute medicine about the guidelines and practicing using them with example cases. Questionnaires were completed before and after the teaching to assess confidence in prescribing opioids. The guidelines were downloaded on the doctors' work phones and laminated on the wards.

Results Junior doctors were not confident in opioid prescribing and adjusting in AKI and Chronic Kidney Disease (CKD) prior to the teaching and availability of new guidelines. Confidence increased after the teaching for prescribing opioids, particularly in patients with renal impairment. For example, confidence in initiating opioids in patients with AKI was on average 5.63 out of 10 before the teaching ( 1 not confident and 10 very confident) and 7.93 afterwards.

Conclusions The availability of specific guidelines for prescribing and adjusting opioids in acute medical admissions for patients with renal impairment significantly increased junior doctors' confidence in this. A repeat audit will now be conducted to determine if this has impacted on the prescribing of opioids in practice. Results of this will be available at the conference. 